\title{
The clinical significances of the abnormal expressions of Piwill and Piwil2 in colonic adenoma and adenocarcinoma
}

This article was published in the following Dove Press journal:

OncoTargets and Therapy

27 May 2015

Number of times this article has been viewed

\author{
Hai-Ling Wang' \\ Bei-Bei Chen' \\ Xin-Guang Cao' \\ Jin Wang ${ }^{2}$ \\ Xiu-Feng $\mathrm{Hu}^{\prime}$ \\ Xiao-Qian Mu' \\ Xiao-Bing Chen' \\ 'The Affiliated Cancer Hospital \\ of Zhengzhou University, Henan \\ Cancer Hospital, Zhengzhou, People's \\ Republic of China; ${ }^{2}$ The First Affiliated \\ Hospital of Zhengzhou University, \\ Zhengzhou, People's Republic of \\ China
}

Objective: The objective of the present investigation was to study the clinical significances of the abnormal expressions of Piwill and Piwil2 protein in colonic adenoma and adenocarcinoma.

Methods: This study had applied immunohistochemical method to detect 45 cases of tissues adjacent to carcinoma (distance to cancerous tissue was above $5 \mathrm{~cm}$ ), 41 cases of colonic adenoma and 92 cases of colon cancer tissues, and their Piwill and Piwil2 protein expression levels.

Analysis: The correlation of both expression and its relationship with clinicopathological features of colon cancer was analyzed.

Results: Positive expression rates of Piwill in tissues adjacent to carcinoma, colonic adenoma, and colon cancer were $11.1 \%(5 / 45), 53.7 \%(22 / 41)$, and 80.4\% (74/92), respectively; the expression rates increased, and the comparisons between each two groups were statistically significant $(P<0.05)$. In each group, the positive expression rates of Piwil2 were $24.4 \%$ ( $11 / 45$ cases $), 75.6 \%$ (31/41 cases), and 92.4\% (85/92 cases); expression rates increased, and the comparisons between each two groups were statistically significant $(P<0.05)$. Piwill expression and the correlation of the degree of differentiation, TNM stage, and lymph node metastasis were statistically significant $(P<0.05)$. Piwil2 expression and the correlation of the degree of differentiation, tumor node metastasis (TNM) stage, and lymph node metastasis had no statistical significance $(P>0.05)$. In colon cancer tissue, Piwil1 and Piwil2 expressions were positively correlated $(r=0.262, P<0.05)$.

Conclusion: The results showed that the abnormal expression of Piwill and Piwil2 might play an important role in the process of colon cancer development.

Keywords: colon cancer, colonic adenoma, Piwil1, Piwil2, immunohistochemical

\section{Introduction}

Colon cancer is becoming a killer and seriously threatens people's health, and the incidence of colon cancer in People's Republic of China has increased significantly in recent years. Compared to that of the European countries and America, the earlyonset age of colon cancer in People's Republic of China obviously has become younger, and the early diagnosis rate is also low. Early colon cancer onset is hidden, often without obvious clinical symptoms, but the illness develops slowly; most of the patients begin to see a doctor after realizing the symptoms such as purulent blood, and abdominal pain, emaciation, and anemia develop after they began to see a doctor. At that time, the disease would have developed to medium-late stage. The current clinical treatment mainly includes chemotherapy, surgery, and targeted therapy, but its function is limited, and 5-year survival rate is low, although it is well known and enshrined in modern guidelines for the treatment of the colorectal cancer that surgery is the first and primary method proven in resectable cancer, especially in clinical stage III. The occurrence and development of colon cancer are related to many genes,
Correspondence: Xiao-Bing Chen The Affiliated Cancer Hospital of Zhengzhou University, Henan Cancer Hospital, 127 Dongming Road, Zhengzhou City, 450008, Henan Province, People's Republic of China Email hailingwangwhl@।63.com 
multiple stages, and mutual interaction of multiple factors. The current study found that the occurrence of colon cancer was related to genetics, lifestyle, environmental pollution, and many other factors. Colonic adenoma is considered as precancerous lesions of colon cancer. Most of the colon cancer develops through hyperplasia, adenoma, and canceration process. Early detection and removal of the colonic adenoma could reduce the incidence of colon cancer. Piwi proteins are tumor stem cell factors. Piwill and Piwil2 belong to Piwi protein family. The current research suggested that cancer stem cells were the origin of the tumor, and determined the occurrence, development, and recurrence of the tumor. The study mainly researched on abnormal expressions of Piwill and Piwil2 in colonic tumor and colon cancer, and explored their function in the occurrence and development of colon cancer, in order to provide certain theoretical basis for clinical treatment.

\section{Materials and methods Materials}

This study selected the colon cancer cases from July 2010 to July 2012, in the pathology department of the First Affiliated Hospital of Zhengzhou University. There were a total of 178 cases of colon adenoma specimens, which were removed under electronic colonoscopy (excluding local canceration and inflammatory polyp) and were archived as wax blocked. In 92 cases of colon cancer specimens, there were 51 cases of male, and 41 cases of female, aging 34-79, with a median age of 56.5 years. The study applied 17 th edition of TNM staging system from the American Joint Committee on Cancer, published in 2010. The cases were divided as follows: 16 cases for stage I, 33 cases for stage II, 31 cases for stage III, and 12 cases for stage IV. According to the degree of differentiation, there were 31 cases of high differentiation, 39 cases of moderate (medium) differentiation, and 22 cases of low differentiation. There were 40 cases with lymph node metastasis, 52 cases with no lymph node metastasis, and 41 cases with colon adenoma and atypical hyperplasia. In the control group, there were 45 cases and above from the tissue adjacent to carcinoma with a distance above $5 \mathrm{~cm}$. All the patients were with complete data, without records of having tumor in other area, and had not received radiotherapy, chemotherapy, and immunotherapy before tumor surgery or endoscopic treatment.

\section{Reagents}

Piwill and Piwil2 rabbit antihuman polyclonal antibodies were bought from Saierbio, LLC, Tianjin, People's Republic of China. The ready-to-use ldpe-g-nvp test kits (biotin) and diaminobezidin (DAB) chromogenic agent were bought from Beijing Zhongshan Golden Bridge Biotechnology Co, Ltd, Beijing City, People's Republic of China.

\section{Methods}

Specimens were fixed with 10\% formaldehyde, and were conventional paraffin embedded, made into $4 \mu \mathrm{m}$ thick serial sections, regularly dewaxed, and repaired of high temperature and high pressure. Specific steps were carried out in strict accordance with the kit instructions. Piwill and Piwil2 were resistant to the concentration of DAB chromogenic agent and wood grain in the ratio of 1:100 after dyeing. Phosphatebuffered saline was treated as the positive control.

\section{Inclusion criteria}

With reference to the literature, Piwil and Piwil2 proteins were mainly expressed in cytoplasm and nuclei. Positive expression is confirmed by yellow color or tan. The positive expression is observed using an optical microscope under a magnification of $\times 400$; five horizons were randomly selected from each section, and each view had a count of 100 cells. According to the score-staining intensity, points were assigned as follows: 0 point for colorless, 1 point for light yellow, 2 points for tan, 3 points for brown. According to the positive cell percentage of scoring, 0 point was assigned for positive cells less than $5 \%, 1$ point for positive cells between $5 \%$ and $24 \%, 2$ points for positive cells between $25 \%$ and 49\%, 3 points for positive cells between $50 \%$ and $74 \%$, and 4 points for positive cells being $75 \%$ or higher. The level of expression was decided by the staining intensity score with positive cells percentage: $0-1$ point for negative (-), 2-3 points for weakly positive $(+), 4-5$ points for positive $(++)$, and 6-7 points for strong positive $(+++)$. Immunohistochemical staining was performed by two highly qualified pathologists under double-blind conditions.

\section{Statistical methods}

Data SPSS17.0 package was adopted to improve the analysis, and comparisons between the test groups were performed using $\chi^{2}$ analysis. The study of correlation between Piwill and Piwil2 proteins adopted the Spearman correlation analysis, with inspection level at $a=0.05$.

\section{Results}

(1) There was positive expression of Piwill and Piwil2 proteins in cancer, colonic adenoma, and colonic carcinoma tissue (Figures 1-6). Piwil1 protein in cancerous tissue 


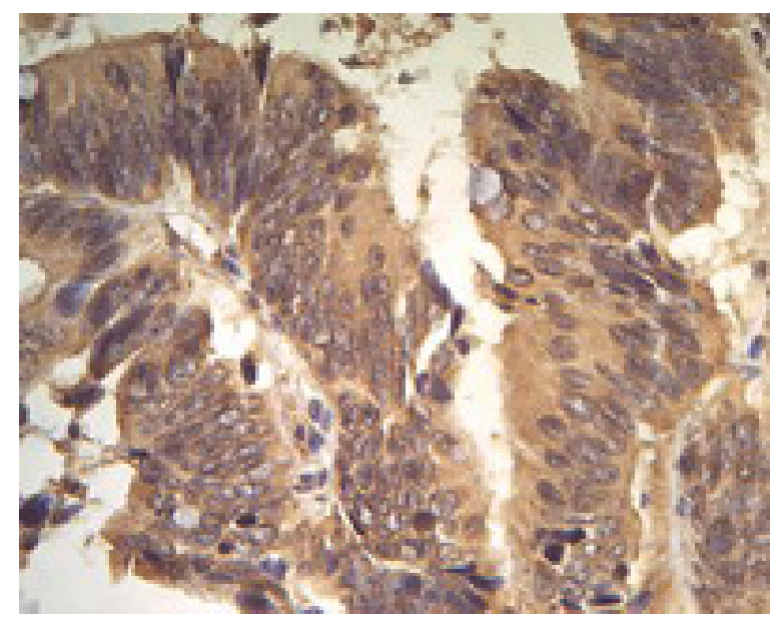

Figure I Positive expression of Piwill in colon cancer, $\times 400$.

compared to tissue adjacent to carcinoma: $\chi^{2}=59.488$, $P=0.000$; comparison between the cancer and adenoma tissue: $\chi^{2}=10.126, P=0.001$; adenoma tissue compared with tissue adjacent to carcinoma: $\chi^{2}=18.031, P=0.000$. In cancerous tissue, the results were as follows: Piwil2 protein compared to tissue adjacent to carcinoma: $\chi^{2}=66.528$, $P=0.000$; comparison between the cancer and adenoma tissue: $\chi^{2}=7.164, P=0.007$; adenoma tissue compared with tissue adjacent to carcinoma: $\chi^{2}=22.477, P=0.000$ (Table 1).

(2) For the relationship between positive expression of Piwill and Piwil2 proteins and clinicopathological factors of colon cancer, see Table 2 .

(3) There were 71 cases of positive expression of Piwill and Piwil2 in colorectal tissues, four cases were negative, and both the expressions were significantly positively correlated $\left(r=0.262, \chi^{2}=6.798, P<0.05\right)$.

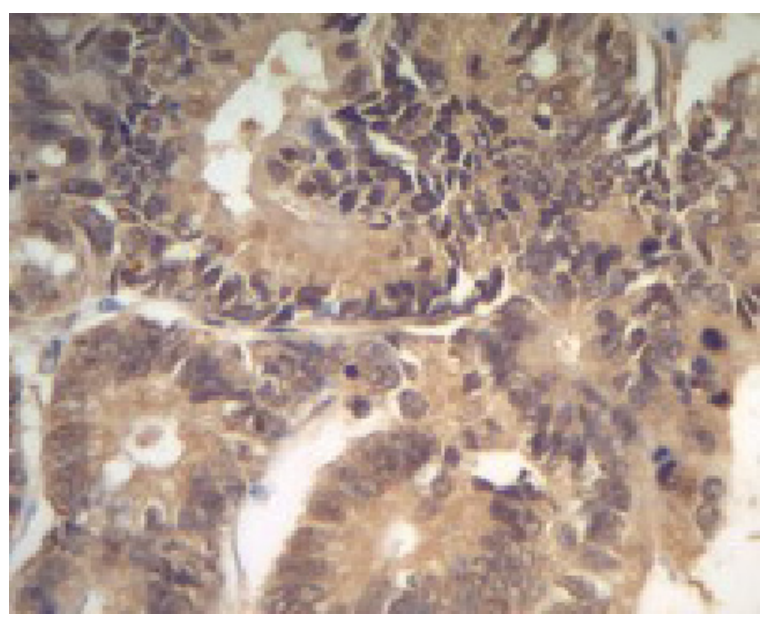

Figure 2 Positive expression of Piwil2 in colon cancer, $\times 400$.



Figure 3 Positive expression of Piwill in colonic adenoma, $\times 400$.

\section{Discussion}

In recent years, the study found that in a wide variety of tumor tissues, only a small part of the tumor cells were capable of self-renewal and proliferation differentiation, which are named as tumor stem cells, and most of the cancer cells do not have this ability. Cancer stem cells occupied a very small proportion of cancer cells in tumor tissue, and could only form new tumors, while other tumor cells died after a limited proliferation differentiation. ${ }^{1}$ Cancer malignant degrees were decided by the proportion of cancer stem cells in tumor tissue; the higher the ratio, the greater the malignant degree. ${ }^{2}$ Mutations in tumor stem cells were derived from normal stem cells. Cancer stem cells and normal stem cells were with similar features in many ways; both had the ability of self-renewal, proliferation, and differentiation into a new tissue. Cancer stem cells played an important role in tumor occurrence, invasion, metastasis, and recurrence. ${ }^{3}$



Figure 4 Positive expression of Piwil2 in colonic adenoma, $\times 400$. 


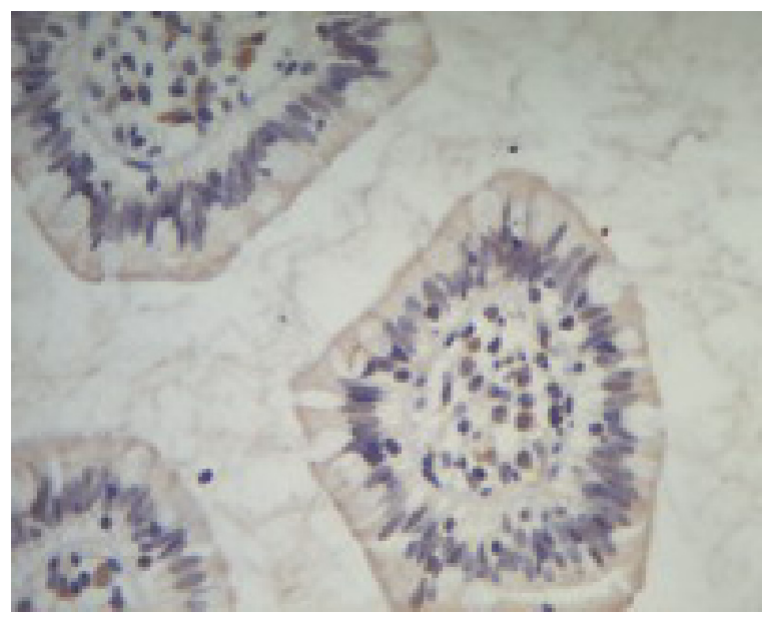

Figure 5 Negative expression of Piwill in para-carcinoma tissue, $\times 400$.

Many cancer stem cells had been isolated from leukemia, breast cancer, brain tumors, and other cancers.

Piwi form was first found by Lin in reproductive cells of fruit flies in the late 1990s, who supposed that it functioned importantly in reproduction and stem cell self-renewal. ${ }^{4}$ Piwi family included Piwill (Hiwi), Piwil2 (his), Piwil3, and Piwil4. Piwi family had two conservative domains, ${ }^{5}$ which were PAZ (Piwi/Argonaute/Zwille) structural domains and Piwi structural domains. Piwi structural domains were composed of 300 amino acids. Piwi family was an important part of microRNAs of RNA-induced silencing complex (RISC). RISC played a key role in two pathways: RNA interference and miRNA inhibition of gene expression. Piwi family had proved to be necessary for sperm formation in mice. Recent studies had confirmed that Piwi family played an important role in stem cell self-renewal, regulation, and control of spermatogenesis and translation, and was the key regulator of stem cells. Decreasing of Piwi protein expression could

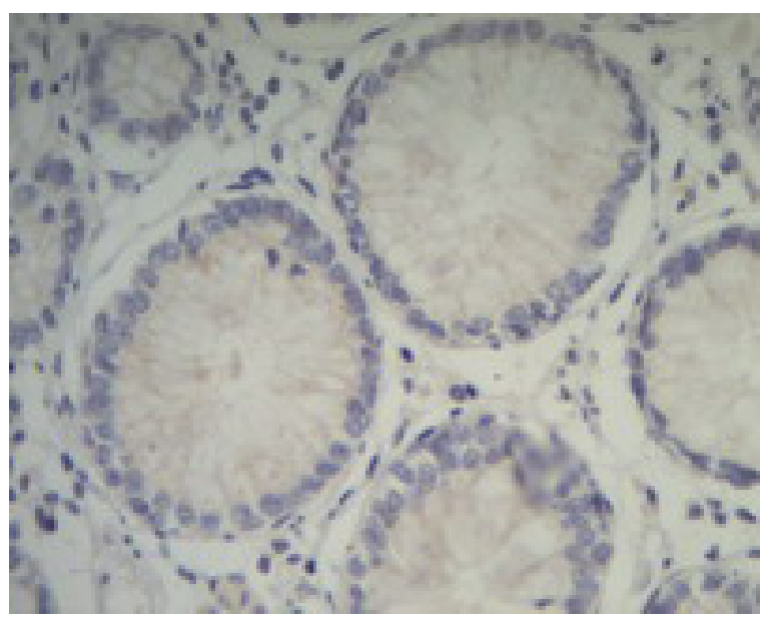

Figure 6 Negative expression of Piwil2 in para-carcinoma tissue, $\times 400$.
Table I Positive expression of Piwill and Piwil2 proteins in cancer, colonic adenoma, and cancerous tissue

\begin{tabular}{llll}
\hline Tissue & $\begin{array}{l}\text { Number } \\
\text { of cases }\end{array}$ & $\begin{array}{l}\text { Piwill-positive } \\
\text { cases (\%) }\end{array}$ & $\begin{array}{l}\text { Piwil2-positive } \\
\text { cases (\%) }\end{array}$ \\
\hline $\begin{array}{l}\text { Tissue adjacent } \\
\text { to carcinoma }\end{array}$ & 45 & $5(\mathrm{II} . \mathrm{I})$ & $\mathrm{II}(24.4)$ \\
Colonic adenoma & $4 \mathrm{I}$ & $22(53.7)$ & $3 \mathrm{I}(75.6)$ \\
Cancerous tissue & 92 & $74(80.4)$ & $85(92.4)$ \\
\hline
\end{tabular}

significantly decrease the rate of stem cell differentiation; excessive expression could increase the quantity of stem cells and rate of differentiation. The lack of human Piwi led to the failure of reproductive stem cells, and decline of embryonic development of Piwi will affect the original germ cell differentiation. Cancer stem cells had the similar characteristics of normal stem cells, with the ability of proliferation, selfrenewal, and differentiation, and Piwi functioned importantly in maintenance of cancer stem cell differentiation.

Piwill had been firstly detected in the human testicles, through fluorescence in situ hybridization. People had found its long arm located on chromosome 12, which is also the position of adult testicular germ cell cancer-related gene. Piwill protein had high expression in seminoma, and no expression in testicular germ cell tumors and non-seminoma. Piwill was also expressed in human hematopoietic stem cells, and was not expressed in differentiated cells. In the study of Liu et $\mathrm{al}^{6}$ it was found that Piwill protein expression in gastric cancer tissue was significantly higher than normal gastric mucosa. They ${ }^{7}$ thought that Piwill expression in esophageal squamous carcinoma was associated with poor prognosis. In this experiment, Piwill in colonic tumor and colon cancer tissues were highly expressed. From the adjacent tissue, carcinoma, and colonic adenoma to colon cancer, expressions were increasing, and comparison between each and the difference were statistically significant. Expression in colon cancer tissue and correlation of tissue differentiation, TNM stages, and lymph node metastasis were statistically significant, and with gland tumor malignant progression, colon cancer malignant degree, the progression in clinical stage, and lymph node metastasis, Piwill protein expressions were correspondingly increasing. This indicated Piwill expression in precancerous lesions of colon cancer; adenoma phase has begun to work. The proliferation, invasion, and metastasis of colon cancer might have an effect to a certain degree.

In case of Piwil2 expression in mice primitive germ cells, its shortage could make spermatocyte unable to form sperm cells. ${ }^{8}$ Piwil2 was located at $1 \mathrm{p}$, which was usually missed in primitive neuroectodermal tumors, Wilms tumor, and many malignant tumors, ${ }^{9}$ suggesting that it may play an important 
Table 2 Relationship between positive expression of Piwill and Piwil2 proteins in colon cancer tissue and its clinicopathological factors

\begin{tabular}{|c|c|c|c|c|c|c|c|}
\hline $\begin{array}{l}\text { Clinical pathological } \\
\text { factors }\end{array}$ & $\begin{array}{l}\text { Number } \\
\text { of cases }\end{array}$ & $\begin{array}{l}\text { Number of Piwill- } \\
\text { positive cases (\%) }\end{array}$ & $\chi^{2}$ & $P$ & $\begin{array}{l}\text { Number of Piwil2- } \\
\text { positive cases (\%) }\end{array}$ & $\chi^{2}$ & $P$ \\
\hline Sex & & & 1.094 & 0.296 & & 0.240 & 0.624 \\
\hline Male & 51 & $43(84.3)$ & & & $46(90.2)$ & & \\
\hline Female & 41 & 31 (75.6) & & & $39(95.1)$ & & \\
\hline Age (years) & & & 0.112 & 0.737 & & 1.487 & 0.223 \\
\hline 55 or below & 39 & $32(82.1)$ & & & $34(87.2)$ & & \\
\hline Above 55 & 53 & $42(79.2)$ & & & $51(96.2)$ & & \\
\hline AJCC 7th edition & & & 5.403 & 0.020 & & 1.950 & 0.163 \\
\hline \multicolumn{8}{|l|}{ TNM stage } \\
\hline I+ II stage & 49 & $35(71.4)$ & & & $43(91.8)$ & & \\
\hline III + IV stage & 43 & $39(90.7)$ & & & $42(93.0)$ & & \\
\hline Degree of differentiation & & & 10.889 & 0.001 & & 0.014 & 0.906 \\
\hline High & 31 & $19(61.3)$ & & & $28(90.3)$ & & \\
\hline Medium or low & 61 & $55(90.2)$ & & & $57(93.4)$ & & \\
\hline Lymph node metastasis & & & 6.546 & 0.011 & & 0.186 & 0.666 \\
\hline No & 52 & $37(7 \mid .2)$ & & & $47(90.4)$ & & \\
\hline Yes & 40 & $37(92.5)$ & & & $38(95.0)$ & & \\
\hline
\end{tabular}

Abbreviation: AJCC, American Joint Committee on Cancer; TNM, tumour node metastasis.

role in the formation of tumors. This study preliminarily applied immunohistochemical experiments to confirm that Piwil2 widely expressed in colon cancer and precancerous lesions of adenoma tissue. From the adjacent tissue, carcinoma, and adenoma to colon cancer, positive expression rate of Piwil2 protein was in the increasing trend. And comparison between the groups has statistical significance. Piwil2 protein expression in the colon, tissue differentiation, TNM stages, and lymph node metastasis has no statistical significance. This was similar with research on other tumors, and preliminarily confirmed that Piwil2 may play a role in the process of colon cancer development in its early stages, but its specific mechanism was not fully clear, and required further research with a large sample at the cellular level and molecular level.

Piwill and Piwil2 protein expression rose in colonic tumor stage, and in colon cancer tissue, Piwil1 and Piwil2 protein expressions were positively correlated, indicating that both proteins may have correlation in the occurrence and development of colon cancer. Piwi-interacting RNA (piRNA) was recently found as a new type of short control RNA in mice spermatogonium. Piwi family proteins mainly functioned through piRNA. From this study, Lee et $\mathrm{al}^{10}$ found that through activating endogenous RNAi mechanism, Piwil2 effected on important cell signaling pathways such as Stat3/Bcl-XL and Stat3/CyclinD1, inhibited cell apoptosis, regulated cell cycle, and performed an important role in the tumorigenesis and development. Pillai et $\mathrm{al}^{11}$ found that Piwil2 might have functioned through combination with target mRNA inhibition of protein synthesis.

At present, most of scholars believe that approximately $80 \%$ of colorectal cancers are developed from adenoma. ${ }^{12}$
In this experiment, high expression of Piwill and Piwil2 had appeared in lesions in the early stages of the colon cancer (adenoma stage), which promotes that their high expression might be an early event of colon cancer.

Nowadays, people could not fully understand the function and regulatory mechanism of Piwi, and its participation in the specific mechanism of tumorigenesis, exact role in the occurrence and development of tumor, and different stages of tumor and the different expressions of different tumors are not entirely clear. While deepening on Piwi and cancer stem cells, the research could also provide a new way to study the origin, biological behavior of tumor, and the treatment against tumor. The target of the treatment of tumor is to remove the cancer cells from the body. The traditional treatment was with surgery, radiation, and chemotherapy and immunotherapy. These methods could only kill mature differentiated cancer cells, and reduce tumor size and number to a certain extent, but they could not kill the tumor stem cells, and cannot give a radical cure effect. Deepening the research on cancer stem cells may have a far-reaching influence on the idea of cancer treatment. Conventional radiation and chemotherapy cannot kill tumor stem cells, and tumor stem cells were more likely to become drug resistant, and not sensitive to radiation therapy. Therefore, treatment against tumor should focus on the purpose of killing cancer stem cells. Only in this way, one can solve the problem of tumor drug resistance, and prevent tumor metastasis. Tumor stem cell theory could change the method of chemotherapy drugs application. It was promising to invent a treatment strategy to kill cancer stem cells. Therefore, the discovery of selective killing of cancer stem cells was our research direction. Tumor treatment 
completion from palliative to radical change needed more in-depth understanding of the tumor.

It has been proposed that Piwill and Piwil2 might be potential biomarkers of colon cancers in human beings. ${ }^{13}$ Furthermore, Piwil2 is widely recognized as a stem cell protein, and it is expressed in various tumors, especially colon cancers, by a mechanism of preventing apoptosis by the Stat3/Bcl-XL pathway. Therefore, Piwill and Piwil2 may play roles in colorectal cancer through the activation of the Stat3/Bcl-XL signaling pathway, which remains to be investigated. In addition, additional experiments are necessary to go beyond the preliminary results shown in order to provide more mechanistic insight into the functional role of Piwill and Piwil2 in colon cancer by small interfering RNA and overexpression techniques.

If expression is observed in cancer-adjacent mucosa to some extent, field carcinogenesis could have occurred in colorectal carcinogenesis, which is not generally observed in the sporadic colorectal carcinogenesis. If the expression of Piwill is related to the tumor stage, differentiation, and lymph node metastasis, Piwill should be related to the prognosis of the colorectal cancer. It is important to investigate the expression levels of different types of precancerous lesions with various malignant potential hyperplastic tubular, villous, and tubulo-villous polyps in future.

\section{Acknowledgments}

This work was supported by the Provincial Science and Technology Innovation Talents Plan of 2014, Establishment of Individualized Treatment Fingerprint for miRNA predicting Colorectal Cancer, and the Medical Science and Technology Key Plan Provincial Established Project of 2011 (number 2011010012).

\section{Authors' contributions}

Hai-Ling Wang was the guarantor of integrity of the entire study. Xiao-Bing Chen was involved in study concepts and study design. Jin Wang was responsible for definition of intellectual content and literature research. Xin-Guang Cao conducted the clinical studies and experimental studies. Bei-Bei Chen was involved in data acquisition and data analysis. Xiao-Qiang Mu performed the statistical analysis and prepared the manuscript. Xiu-Feng Hu carried out the manuscript editing and review.

\section{Disclosure}

The authors report no conflicts of interest in this work.

\section{References}

1. Reya T, Morrison SJ, Clarke MF, Weissman IL. Stem cells, cancer and cancer stem cells. Nature. 2001;414(2):105-111.

2. Sharpless NE, DePinho RA. Telomeres. Stem cells, senescence and cancer. J Clin Invest. 2004;113(2):160-168.

3. Marx J. Cancer research: mutant stem cells may seed cancer. Science. 2003;301:1308-1310

4. Lin H, Spradling AC. A novel group of pumilio mutations affects the asymmetric division of germline stem cells in the Drosophila ovary. Development. 1997;124:2463-2476.

5. Cerutti L, Mian N, Bateman A. Domains in gene silencing and cell differentiation proteins: the novel PAZ domain and redefinition of the Piwi domain. Trends Biochem Sci. 2000;25:481-482.

6. Liu XY, Sun Y, Guo JP, et al. Expression of hiwi gene in human gastric cancer was associated with proliferation of cancer cells. Int J Cancer. 2006;118:1922-1929.

7. He W, Wang ZH, Wang Q, et al. Expression of HIWI in human esophageal squamous cell carcinoma is significantly associated with poorer prognosis. BMC Cancer. 2009;9:426.

8. Kuramochi-Miyagawa S, Kimura T, Ijiri TW, et al. Mili, a mammalian member of piwi family gene, is essential for spermatogenesis. Development. 2004;131(4):839-849.

9. Dome JS, Coppes MJ. Recent advances in Wilms tumor genetics. Curr Opin Pediatr. 2002;14(1):5-11.

10. Lee JH, Schütte D, Wulf G, et al. Stem-cell protein Piwil2 is widely expressed in tumors and inhibits apoptosis through activation of Stat3/ Bcl-XL pathway. Hum Mol Genet. 2006;15(2):201-211.

11. Pillai RS, Artus CG, Filipowicz W. Tethering of human ago proteins to mRNA mimics the miRNA-mediated repression of protein synthesis. RNA. 2004;10(10):1518-1525.

12. Fang J-Y. Pay attention to the treatment of colorectal adenomas resarch. Chin Dig Mag. 2010;30(7):433-435.

13. Lan LI, Chaohui YU, Hengjun G, Youming LI. Argonaute proteins: potential biomarkers for human colon cancer. BMC Cancer. 2010; 10:38.
OncoTargets and Therapy

\section{Publish your work in this journal}

OncoTargets and Therapy is an international, peer-reviewed, open access journal focusing on the pathological basis of all cancers, potential targets for therapy and treatment protocols employed to improve the management of cancer patients. The journal also focuses on the impact of management programs and new therapeutic agents and protocols on

Submit your manuscript here: http://www.dovepress.com/oncotargets-and-therapy-journa

\section{Dovepress}

patient perspectives such as quality of life, adherence and satisfaction. The manuscript management system is completely online and includes a very quick and fair peer-review system, which is all easy to use. Visit http://www.dovepress.com/testimonials.php to read real quotes from published authors. 